\title{
MULTI-SERVICE MANAGEMENT IN A MULTI-PROVIDER ENVIRONMENT
}

\author{
David Lewis and Thanassis Tiropanis
}

University College London, U.K

\section{INTRODUCTION}

As the spread of digital networks makes access to data communications globally available, the interest of communication service providers is switching away from the provision of these bearer networks and towards the provision of the value added services that will operate over them. At the same time the liberalisation of telecommunication markets is precipitating a dramatic change in the profile of communication service providers. As can already be seen in the U.K., this involves both an increase in the number of providers and the specialisation of individual providers in order to exploit market niches. Such a market will be highly dynamic with service offerings and prices changing rapidly. The role of the customer will also change with the proportion of data traffic supporting corporate networks shrinking while that used by both commercial and domestic customers for accessing third party services increases. This will be accompanied by an increase in the number of commercial customers who buy services, not for their own end-users, but to resell in an integrated value added service package to their own customers.

In this complex telecommunications markets the open management, not only of the networks, but of the services themselves will become increasingly important. The large number and diversity of roles of the market players makes the management of inter-organisational relationships fundamentally important to the management of services.

The ITU's series of recommendations on the Telecommunication Management Network (TMN) (1) provides a basis for inter-domain management, however, this and other standards have so far concentrated on the management of individual network components and of networks operated by single organisations. This paper provides an initial example of how the management of multiple services in a complex multi-player market can be modelled using TMN techniques for implementation on existing management platforms. The paper begins by briefly outlining current work in this field before describing aspects of this multi-player multi-service management problem and how they can be modelled and implemented in a real system.

\section{CURRENT WORK}

This section briefly summarises the extent to which service management has been addressed in the ITU's M.3100 recommendation and the work of the Network Management Forum. It then gives a quick overview of work on service management performed in two RACE projects, PRISM and PREPARE.

\section{M.3100 Recommendation}

This recommendation (2) provides a generic information model which is independent of underlying technologies and architectures. It's purpose is to describe the information exchanged between management systems in different domains in order to satisfy certain requirements.

In order to generate those requirements three viewpoints were considered in the design of the information model: the network element level viewpoint, the network level viewpoint and the service level viewpoint. The network element level viewpoint is concerned with the information required to manage a single network element. The network level viewpoint has to do with the information exchanged between many Network Element management systems in order to provide end-to-end connectivity.

The service level viewpoint is concemed with the utilisation of the end-to-end connections provided by the network level management, in order to provide network services.

Though the service level requirements were considered in the design of this information model there is no actual support for management areas such as customer configuration, usage monitoring and billing that are important in service level management. It focuses more on the abstraction of the physical and logical network resources for providing interoperability between the 
management systems of different domains and end-toend connectivity.

\section{Network Management Forum}

The Network Management Forum (NMF) was formed in 1988 by computer systems vendors, service providers. telecommunications equipment vendors and users. It's main purpose is to provide integrated service management over different network management technologies and architectures (i.e. OSI network management, SNMP, CORBA network management). The recommendations this group makes are generated in the form of ensemble documents (3). An ensemble provides a top-down view of a particular solution to a management problem.

The Customer Administration Configuration Management (CACM) ensemble (4) specifies the management interface between an operations system and a telecommunications switching element manager for service provisioning to the customer. The network resources are abstracted so that network provisioning does not have to deal with specific technical implementations.

CACM is a good example of a top-down definition of multi-service provisioning but for very specific and strongly telephony related services. As far as service management is concerned, it focuses more on configuration management of customer information related to the services used by the customer. Aspects concerning performance, billing and security are excluded. A multi-provider environment cannot supported since all the services involved are explicitly specified and only direct service provisioning is considered.

\section{The PRISM Project}

PRISM is a RACE project which aims to investigate service management for the future deregulated telecommunications market in Europe. It has investigated the definition of service management systems following a specific methodology developed by the project. PRISM follows a top-down approach to the problem. The service management systems must be defined in a way that validates the TMN reference configurations specified by PRISM.

Reference configurations define the relationships between the different actors (players) in the service provisioning arena, the TMN reference points used and the TMN interfaces where possible. A specific framework, called the Service Management Reference Configuration (SMRC) (5), was developed for supporting this procedure of validating service.
This SMRC provides abstract architectures, which are independent of any specific platforms, for management services. These abstract architectures can be applied to specific platforms and used to form concrete architectures. A concrete architecture can then be used for the implementation of a management system. A concrete architecture can be viewed as being the equivalent of an NMF ensemble.

The SMRC framework considers five of the ODP viewpoints (6) to ensure that it covers all the aspects of the problem.

- The enterprise viewpoint which is concerned with business and management policies and actor's roles (e.g. provider, customer, etc.). The enterprise model describes the interactions between the actors, integrating security management and covering other requirements such as quality of service.

- The information viewpoint provides a generic view of information resources of the network in accordance with X.720, X.725, TMN and M.3100 standards.

- The computational and engineering viewpoint are considered but to a lesser extent while the technology viewpoint is currently outside the scope of PRISM.

The enterprise viewpoint together with the information viewpoint used in the SMRC abstract architecture provide very good support for a multi-service management in a multi-provider environment. The SMRC is a meta-model for the definition of concrete architecture and therefore it does not provide specific solutions or an information model. However following the SMRC guidelines in PRISM, pan-European VPN and UPT management services have since been defined (7).

Using the SMRC framework it is possible to define more service management systems. Some of them can be customers of some services or providers to others. What is needed is an information model and a description of the information exchanged among those services to support the more complicated functions a deregulated multi-provider and multi-service environment requires.

\section{The PREPARE Project}

The PREPARE project has already been investigating issues of inter-domain management using TMN techniques. This has been based on the implementation of an inter-domain VPN management service over a broadband testbed (8) and a more in depth case study of the requirements for VPN management. The model used in both was of a specialised value added service provider 


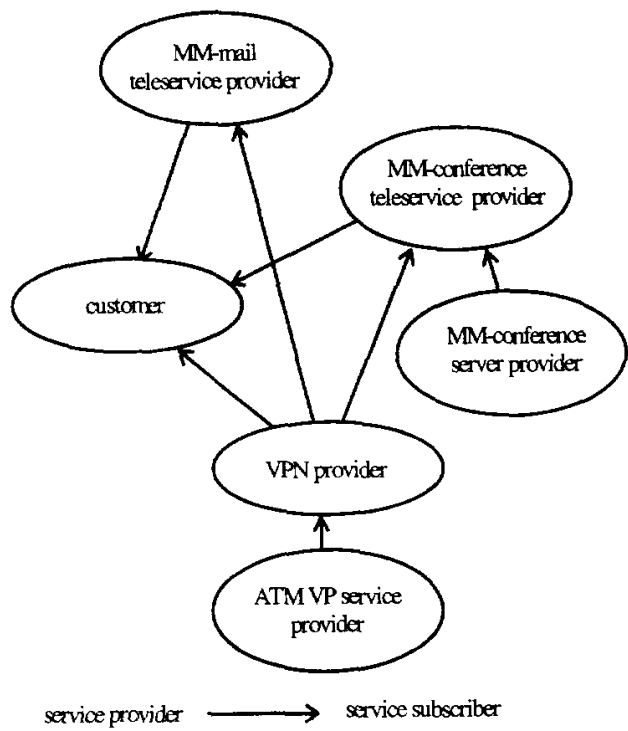

Figure 1.: Actors and provision of services

managing the resources of two separate PN providers in order to provide a VPN service to a multi-site corporate customer. Management of the customer site networks was included in this model so that network resources can be managed in a homogeneous way from end-user terminal to end-user terminal as part of the VPN service.

The case study produced a set of management service features that should be provided by a VPN provider. It also included detailed work on how the sharing of management knowledge between organisations required by such management services could be organised through an integration of X.700 management and X.500 directory services (9).

\section{TMN APPLIED TO SERVICE MANAGEMENT}

The approach chosen for this work was to attempt to analyse and identify some of the key requirements of multi-player multi-service management in the forms of service features, and then to attempt to define information models and manager functions that could be readily implemented and integrated in existing management platforms. It is hoped that this work will achieve at least the following aims; to determine whether TMN principles can be extended effectively to this form of complex service management and to gain further insight into the real requirement of such management by the rapid prototyping and trial use of the TMN systems in a real testbed.
To provide a firm basis for this work a specific enterprise framework was developed. This framework was based on the work already performed in PREPARE but with additional actors being added in order to adequately represent the potential complexities of multiservice management. The actors, all assumed to be separate organisations, and their roles are summarised below:

- Multimedia Mail Teleservice Provider: This actor provides a multimedia mail service to its customers by allowing them access to a multimedia message storage server. Network access between the customer sites and the server is provided by a VPN service that this actor subscribes to from a separate VPN provider

- Multimedia Conference Server Provider: This actor provides access to a multimedia conferencing server that supports multiplexing of multimedia data streams over different network technologies, e.g. ATM, Basic Rate ISDN, Internet.

- Multimedia Conferencing Teleservice Provider: This actor provides a multimedia conferencing teleservice by integrating and re-selling the service it buys from the multimedia conferencing server provider and the VPN provider.

- VPN provider: This actor provides an end-to-end VPN service, both to customers wishing to interconnect their corporate network sites and to the teleservice providers described above for integration into their service offerings.

- VP service providers: This actor is a public network operator (PNO) that provides a ATM virtual path service across its own and other PNO domains.

- Customer: this actor is an organisation that subscribes separately to the VPN service, the multimedia mail teleservice and the multimedia conferencing service in order to allow access to these service between end users at several remote corporate sites.

The enterprise also includes multiple VPN providers which co-operate to shared management information on specific customers, and multiple VP service providers which co-operate in order to offer the VP service over a wider geographical area. The resulting model of service relationships is shown in figure 1 .

To analyse the management requirements of this specific model a set of scenarios was generated to demonstrate various aspects of service management, including service provisioning, service configuration, fault management, performance management, accounting management and service termination. This analysis revealed that the model of service management 
relationships between the players was more complicated than just that between service subscriber and service provider. This partially resulted from the premise that management services should be accessed directly from the player most suited to providing that service. For example an end customer wishing to access purely VPN related management information related to a VPN service used as part of a multimedia teleservice subscription should be able to obtain this service directly from the relevant VPN provider rather than through the teleservice provider's management system. This reduces the need for sharing management information between hierarchically related providers thus avoiding the concomitant overhead of maintaining this information's consistency. The relationship between the consumer of a service and its supplier can be split into two types. The first where there is a direct commercial relationship between the consumer (customer) of the service and its supplier (provider). The second is where there is no such direct commercial relationship and payment of the service is made from consumer to supplier though resale via one or more third parties. Thus we can categorise the roles played by any organisation for any specific service instance to be either direct customer or provider for the first type of relationship, or third party customer or provider for the second type.

It seems clear from this analysis of service relationships in a specific enterprise model of that design of information models for the TMN systems required by each player to manage these would benefit from being based fundamentally on the identification of information related to a specific service instance. This is essential where third party customer/provider relationships existed so that the customer could identify the portion of the management information tree related to any specific service instance it had subscribed to, and so that the provider could identify the customer (not necessarily the one accessing the management information) paying for the service instance fro accounting, security and possible fault management functions.

A simple managed object (MO) was therefore introduced for this purpose. This is intended to act as the head of a containment sub-tree housing all the MOs concerned with this service instance in both the customer and provider agents. It possesses a single minimum attribute that represents a service instance registration identifier. This identifier is allocated by the provider at the top of the service re-sale hierarchy, to which the end customer subscribes directly for a service instance. Such an identifier could therefore consist of a field that uniquely identifies the provider organisation (this would require some form of centralised administration) and a service instance field administered by that organisation. This identifier is then passed to any providers employed by the customers direct provider in order to identify the service instance MO heading the containment tree for that service instance. This structure can then be applied recursively to other providers that this third party provider subscribes to and which contains management information that the end customer should be able to access.

The hierarchical nature of each MO tree can be viewed as explicitly identifying the direct customer responsible for paying for the service instance, while the service registration identifier allows both direct and third party customer to easily locate the base of the management information tree related to a particular service instance by using the base object filter feature of the CMIP protocol. This location of management information can be further simplified by using the integration of X.700 and X.500 Directory to provide a global naming tree for service instance MOs. The direct service provider to the end customer for a particular instance can set up a directory object that contains links to all the service instance MOs in its own and other provider agents with the same service registration identifier. This will provide the customer with what appears to be a single information tree containing all the MOs related to the service instance while at the same time hiding the distribution of these MOs over different provider's agents as well as obscuring other management information in those agents.

\section{FURTHER WORK}

Though no formal description of services will be under taken in this work, it is felt that this information model and the associate manager functions provide the basis of a mandatory service instance management service feature that should be employed in all TMN-based service management systems. Work is currently proceeding to define the information models for the individual services with the VPN and Global Store information's models being nearest completion due to previous work in this area. It is hoped, however, that these management services can be divided into further clearly identifiable management service features, and that service features for common management functions can be identified so that implementations of the relevant MOs and manager functions can be reused in the various management systems. Currently, however, no clear methodology exists to guide this process so a parallel activity is both tracking this work while trying to use the experience gained to provide insight into the integration of IN and TMN techniques. 
The TMN systems outlined here will be implemented on a variety of platforms, i.e., Hewlett-Packards OpenView, IBM's TMN 6000 , Ericsson's TMOS and UCL's OSIMIS. The resulting service level operations system will be integrated with network and network element operations system and used to manage resources on an ATM testbed linking ATM LANs and multimedia servers in London, Berlin and Copenhagen. The network components of the testbed will be operational by quarter of 1995 while it is hope to demonstrate the operation of this service management testbed in concert with network resource management during the last quarter of 1995.

\section{CONCLUSION}

An analysis has been conducted into the problems involved in managing multiple service in the multiple provider environment of a future open service market and to what technologies are currently available to support this. TMN technology appears to be the only one available with existing platforms that are widely accepted in the telecommunication industry. Standardisation efforts have, however, so far been directed at the network element and network level. This paper therefore presents a novel application of TMN principles to service management that can be realised in the near term. By basing the structure of the service level management information tree on the concept of the service instance, this scheme fundamentally and explicitly represents the key aspect of service management, i.e. that a service is provided by one organisation to another in exchange for payment, either directly or via some third party. As has been the case in previous work by this group, the service management concepts described here will be implemented on various network management platforms and will be exercised managing trial services over a real testbed.

\section{ACKNOWLEDGEMENTS}

The work described here is funded by the Commission of the European Union under the RACE II project PREPARE (contract number R2004). The views expressed here do not necessarily those of the PREPARE consortium.

\section{REFERENCES}

1. ITU, "Principles for a TMN", Recommendation M.3010

2. ITU, "Generic Network Information Model", Recommendation M.3100
3. Network Management Forum, 1994, "Domain Framework: a general model and Criteria for Ensemble Selection"

4. Network Management Forum, 1994, "Telecommunications Switching Customer Administration Configuration Management, Final Draft"

5. PRISM Deliverable 4, 1993, "Service Management Reference Configuration", Deliverable No: R2041/GMD/WP3/DS/R/004/a3

6. ITU, 1993, "Basic Reference Model of Open Distributed Processing-Part 1 Overview and Guide to Use", X.901 Draft Recommendation

7. PRISM Deliverable 7, 1994, "VPN and UPT Services Management: Second Case Study Report, Vol 1,2 and 3", Deliverable No: R2041/SAL/WP4/DS/R/007/bl

8. Lewis D., Kirstein P., 1994, "A testbed for the Investigation of Multimedia Services and Teleservice Management", Proceeding of the 3rd International Conference on Broadband Islands.

9. Bjerring L., Tschichholz, 1994, "Requirements of Inter-Domain Management and their Implications for TMN Achitecture and Implementation", Proc. of 2nd RACE IS\&N Conference. 